\title{
Feasibility of implementing a national lung cancer screening program: Interim results from the Korean Lung Cancer Screening Project (K-LUCAS)
}

\author{
Jaeho Lee ${ }^{1}$, Yeol Kim ${ }^{1,2}$, Hyae Young $\mathrm{Kim}^{3}$, Jin Mo Goo ${ }^{4}$, Juntae Lim ${ }^{1,2}$, Choon-Taek Lee ${ }^{5}$, \\ Seung Hun Jang ${ }^{6}$, Won-Chul Lee ${ }^{7}$, Chan Wha Lee ${ }^{3}$, Kui Son Choi ${ }^{1,2}$, Boyoung Park ${ }^{1,2}$, Duk Hyoung Lee ${ }^{1}$; \\ for the K-LUCAS Trial Investigators
}

\begin{abstract}
${ }^{1}$ National Cancer Control Institute, National Cancer Center, Goyang, Korea; ${ }^{2}$ Department of Cancer Control and Population Health, Graduate School of Cancer Science and Policy, National Cancer Center, Goyang, Korea; ${ }^{3}$ Department of Diagnostic Radiology, National Cancer Center, Goyang, Korea; ${ }^{4}$ Department of Radiology, Seoul National University College of Medicine, Seoul, Korea; ${ }^{5}$ Division of Pulmonology and Critical Care Medicine, Department of Internal Medicine, Seoul National University College of Medicine, Seoul, Korea; ${ }^{6}$ Department of Pulmonary, Allergy and Critical Care Medicine, Hallym University Sacred Heart Hospital, Anyang, Korea; ${ }^{7}$ Department of Preventive Medicine, College of Medicine, the Catholic University of Korea, Seoul, Korea

Contributions: (I) Conception and design: Y Kim; (II) Administrative support: Y Kim; (III) Provision of study materials or patients: Y Kim; (IV) Collection and assembly of data: All authors; (V) Data analysis and interpretation: All authors; (VI) Manuscript writing: All authors; (VII) Final approval of manuscript: All authors.

Correspondence to: Yeol Kim, MD, PhD. National Cancer Control Institute, National Cancer Center, 323, Ilsan-ro, Ilsandong-gu, Goyang 10408, Korea. Email: drheat@ncc.re.kr.
\end{abstract}

Background: Lung cancer screening conducted in high-risk group using low-dose computer tomography (LDCT) has been reported as an effective method to reduce lung cancer mortality in two large randomizedcontrol trials. However, the effectiveness is uncertain when lung cancer screening is expanded to a nationwide population-based program.

Methods: The Korean Lung Cancer Screening Project (K-LUCAS) is a single-arm cohort study that was conducted from February 2017 to evaluate the feasibility of implementing an organized national lung cancer screening program in Korea. High-risk population aged 55-74 years with more than a 30-pack-year smoking history was recruited. Smoking history was obtained from administering questionnaires at national health screening programs or public smoking cessation programs which are already established programs in Korea. The screening results were reported using the Lung Imaging Reporting and Data System (Lung-RADS), suggested by the American College of Radiology. K-LUCAS was performed by a network-based diagnosis supporting system using a computer-aided detection (CAD) program to maintain screening quality. Current smokers were provided with mandatory smoking counseling.

Results: Among 71,829 participants aged 50 years or older in the national health screening program, $5,975(8.3 \%)$ were eligible for lung cancer screening. Among them, 1,062 (17.8\%) refused to participate in K-LUCAS. Additionally, 779 participants were recruited in the smoking cessation program. Thus, a total of 5,692 eligible high-risk participants were recruited in this study. Among them, 865 (15.2\%) had positive screening results, which requires a further examination; 529 (9.3\%) had Lung-RADS category 3 (indeterminate), and 336 (5.9\%) had category 4 (suspicious of lung cancer); 42 (0.7\%) had confirmed lung cancer. Approximately 66.7\% had early-stage lung cancer: 24 (57.1\%), stage I and 4 (9.5\%), stage II. Six $(1.1 \%)$ patients developed complications at the time of diagnosis, including one death. The anxiety level related to cancer screening was low. Participation in screening encouraged motivation to quit smoking.

Conclusions: K-LUCAS provided promising evidence supporting the implementation of a national lung cancer screening program to detect early stage lung cancer and promote smoking cessation for participants in Asian population. 
Keywords: Lung cancer; screening; low-dose computer tomography (LDCT); smokers

Submitted May 27, 2020. Accepted for publication Dec 11, 2020.

doi: $10.21037 /$ tlcr-20-700

View this article at: http://dx.doi.org/10.21037/tlcr-20-700

\section{Introduction}

Lung cancer is the leading cause of cancer death; 5 -year survival rates for lung cancer ranged from $10 \%$ to $20 \%$ in most countries, without remarkable improvement over the past decade (1). In Korea, lung cancer is the fourth most common cancer, but it is also the most common cause of cancer death with about $28 \%$ of 5 -year survival rate which is still low (2).

In 2011, the National Lung Cancer Trial (NLST) in the United Sates reported that lung cancer screening conducted in a high-risk group with more than 30-packyear smoking history reduced the lung cancer mortality by $20 \%$ (3). More recently, a Dutch-Belgian trial (NELSON) also reported similar results of mortality reduction by lung cancer screening, although the statistical significance was not reported in women (4). Many countries considered implementing a population-based lung cancer screening program. However, the feasibility and effectiveness of lung cancer screening on a population-based scale has been barely evaluated, and may have different results for different countries.

In 2016, the Korean government planned to implement a national lung cancer screening program using low-dose computer tomography (LDCT) to tackle the lung cancer mortality. K-LUCAS is a population-based nationwide prospective trial, which was conducted to evaluate the feasibility of implementing an organized national lung cancer screening program in Korea. The purpose of K-LUCAS is to examine the feasibility of implementing a nation lung cancer screening program in Korea. K-LUCAS is a 2-year trial that was conducted from February 2017 to December 2018. Here, we report the interim results of baseline screening until December 2017 in order to provide an evidence to begin a discussion on implementing a national lung cancer screening (ClinicalTrials.gov number, NCT03394703). We present the following article in accordance with the STROBE reporting checklist (available at http://dx.doi.org/10.21037/tlcr-20-700).

\section{Methods}

The study conformed to the provisions of the Declaration of Helsinki (as revised in 2013). K-LUCAS was approved by the institutional review board of each participating hospital. An informed consent form was obtained from all participants after providing them with information regarding the benefits and harms of lung cancer screening.

\section{Feasibility categories for implementing a national lung cancer screening program}

The multidisciplinary expert committee who conducted the K-LUCAS study approved the five feasibility categories that will be used to evaluate the need for implementing a national lung cancer screening program (Figure 1).

First, we examined if the targeted eligible high-risk individuals would be included in the lung cancer screening program when offered at low cost or free as would be in a national screening program. We tested the reliability of recruiting eligible high-risk smoking history population using questionnaires, which were submitted in national health screening programs or public smoking cessation programs. National health screening programs in Korea provide cardiovascular risk factor screening including blood pressure, fasting blood glucose and cholesterol level, and cancer screening targeting stomach, colon, liver, breast and cervix cancers. Public smoking cessation programs provide counselling and pharmacotherapy for quitting smoking by free or very low cost. We assessed the changes in the proportion of former smokers' participation after the release of public advertisements as willing nontarget low-risk individuals are most likely to be enrolled in the study as former smokers. We started advertising K-LUCAS in newspapers, in public transports, and on notice boards in public offices from October 2017 onward. The advertisement provided information about the benefits and harms of lung cancer screening. The information about the benefits and harms of lung cancer screening 


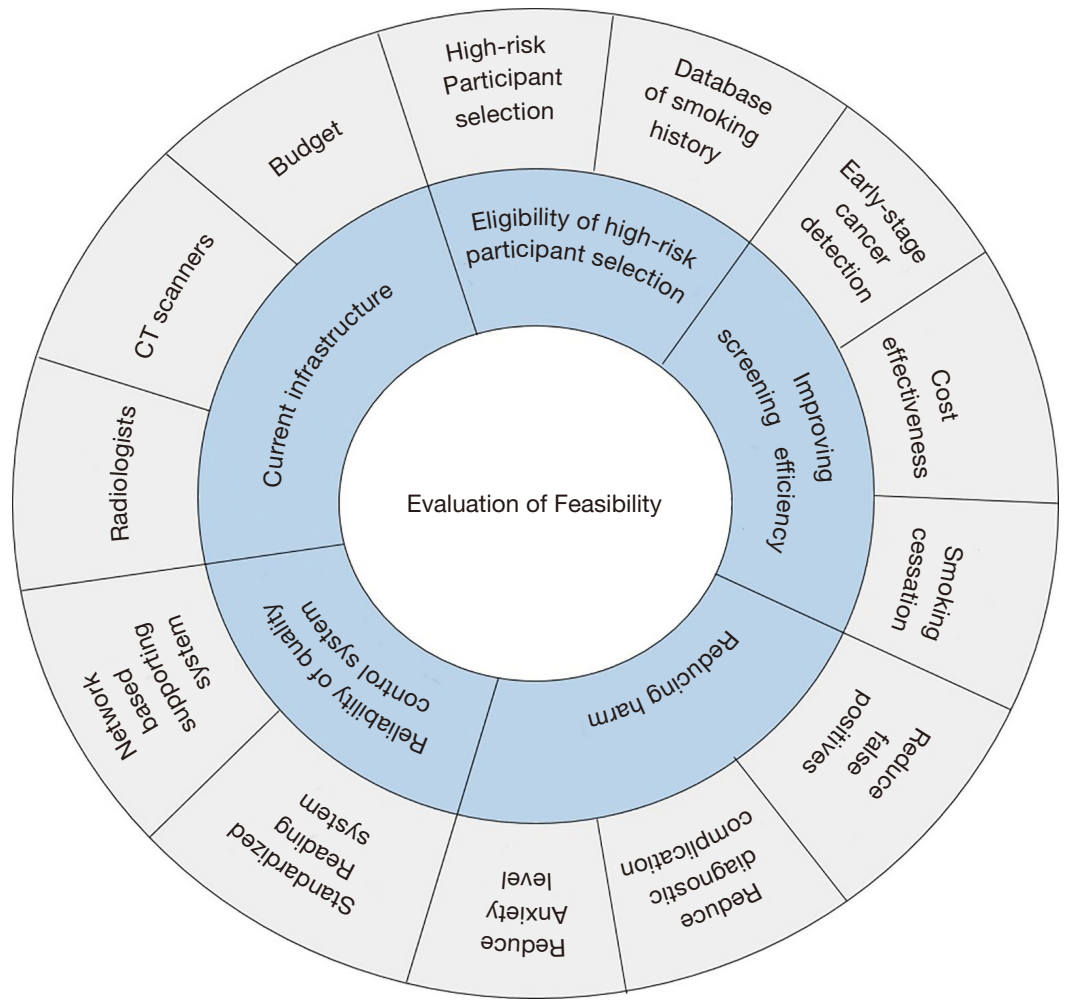

Figure 1 Categories of evaluating feasibility for implementing a nationwide population-based lung cancer screening program.

was also provided to the participants before obtaining the consent of participation in K-LUCAS. The benefits included the possibility of early-stage lung cancer detection and treatment. The harms included the possibility of unnecessary radiation exposure and requirement for invasive confirmatory examination after screening.

The second feasibility category is the screening efficiency. We evaluated if it is feasible to improve the screening efficiency on a national scale in three perspectives: early stage cancer detection, cost-effectiveness, and the effectiveness of smoking cessation intervention.

The early-stage lung cancer detection is defined as lung cancers detected at Stage 1 or Stage 2 based on the $8^{\text {th }}$ edition of the TNM staging system (5). Its rates are compared with those of in the Korean cancer registry as well as with those of the NLST. The cost-effectiveness and full discussion of the smoking intervention in K-LUCAS will be reported separately in the future. Here, we only reported the effect of smoking intervention on participant's motivation to quit smoking. Participant's motivation to quit smoking was measured in terms of their willingness to quit smoking: "Please specify your willingness to quit smoking on a scale of $0-10$. A score of 0 indicates that you have no intention to quit smoking, while a score of 10 indicates that you are very willing to quit smoking."

Third, we evaluated the screening harms in three perspectives: the false positive rates, the number of complications during confirmative diagnosis after screening, and the impact of screening on participant's anxiety about having a lung cancer. The false positive rates and complications at the time of diagnosis were compared with those reported in the NLST. Psychological anxiety from participating in lung cancer screening was measured in terms of the degree of anxiety regarding the possibility of developing a lung cancer: "Please specify your degree of anxiety about lung cancer on a scale of $0-10$. A score of 0 indicates you do not worry at all, and a score of 10 indicates that you worry a lot." We also determined whether participating in lung cancer screening caused an increase in anxiety level or not.

Fourth, we evaluated the capability to control the screening quality. In particular, K-LUCAS has adopted two systematic approaches in pursuit of a better quality control. First, the LDCT screening results were evaluated by radiologists using the standardized Lung Imaging Reporting 
and Data System (Lung-RADS) (6,7). Lung-RADS category 1 is defined as negative; 2, benign appearance; 3 , indeterminate (probably benign); and 4 , suspicious for lung cancer. K-LUCAS also implemented a network-based diagnosis supporting system in pursuit of better quality control. CT images were sent to the cloud system wherein the images were accumulated. These images were initially evaluated using a computer-aided detection (CAD) program in the cloud system. The radiologists from the screening units edited the results using CAD and determined the final Lung-RADS category in the system. K-LUCAS evaluated whether the adoption of network-based quality control system is reliable and can help control the screening quality. This report only briefly discussed on the capability and the screening outcomes using a network-based diagnosis supporting system, but its effectiveness on screening quality will be discussed in detail in a separate report.

Last feasibility category is the level of current infrastructures to implement a national lung cancer screening program. This includes the number of radiologists, number of qualified CT scanners, and the budget. The capacity of current infrastructures in Korea is not evaluated in full detail in this report but only discussed briefly.

\section{Trial design}

K-LUCAS is a single-arm cohort study that was conducted in 14 general hospitals nationwide from February 2017 to December 2018. The trial was conducted in accordance with the original protocol reported previously $(8,9)$. K-LUCAS was approved by the institutional review board of each participating hospital. The authors are responsible of the accuracy and completeness of the data.

K-LUCAS targets high-risk smokers or former smokers (who quit smoking for less than 15 years) aged 55-74 years with an at least 30-pack-year smoking history. Patients (I) with past history of lung cancer; (II) who were unable to move without assistance; (III) who were on treatment for tuberculosis, pneumonia, and interstitial lung disease; (IV) who were diagnosed with cancer within 5 years (exception: thyroid cancer and skin cancer); and (V) who underwent chest computer tomography within 6 months were excluded.

Visitors who participated in the national health screening programs or smoking cessation clinics were recruited. The visitors were required to complete a series of questionnaires, which included questions on smoking history, medical history, and health conditions. Eligible screening candidates were selected based on their responses to the questionnaires.
Selected candidates were asked to participate in the screening via a phone call. An informed consent form was obtained from all participants after providing them with information regarding the benefits and harms of lung cancer screening.

Two additional surveys were conducted to evaluate the participants' level of satisfaction with the lung cancer screening process, the psychological harms of screening, and the motivation for quitting smoking. One survey was conducted on the day of screening. The other was conducted after receiving the screening results. All current smokers underwent the urine cotinine test to verify their current smoking status, and they were surveyed to investigate the change of smoking status by telephone in 6 months after screening. Smoking cessation counseling by a physician was provided mandatorily after screening to currently smoking participants when participants revisit the screening hospital for counselling screening results.

All participants underwent baseline lung cancer screening by LDCT, and the results were standardized by LungRADS $(6,7,9)$. Diagnostic follow-up was also standardized. Participants with negative results (category 1 or 2) were recommended to undergo the next screening within 12 months. Participants with category 3 were recommended to undergo a follow-up examination using LDCT within 6 months. Category 4, suspicious of lung cancer, is divided into $4 \mathrm{~A}, 4 \mathrm{~B}$ and $4 \mathrm{X}$. Participants with category $4 \mathrm{~A}$ were recommended a follow-up examination within 3 months and category $4 \mathrm{~B}$ and $4 \mathrm{X}$ were referred to a pulmonology specialist for diagnosis. This study reported the results of the follow-up examination for 15 months (until December 2018) at maximum. Informed consent was obtained before participation in the follow-up examination, and the medical records in all participating hospitals were reviewed.

Those participants who did not undergo a followup examination within the recommended interval were contacted by telephone (a minimum of three attempts for non-responders) and were urged to undergo further evaluation.

\section{Statistical analysis}

The association between public advertisements and participant characteristics was evaluated using the chisquare test. Changes in the participants' level of anxiety associated with the development lung cancer and in their willingness to quit smoking before and after lung cancer screening were evaluated using a paired t-test. Smoking 


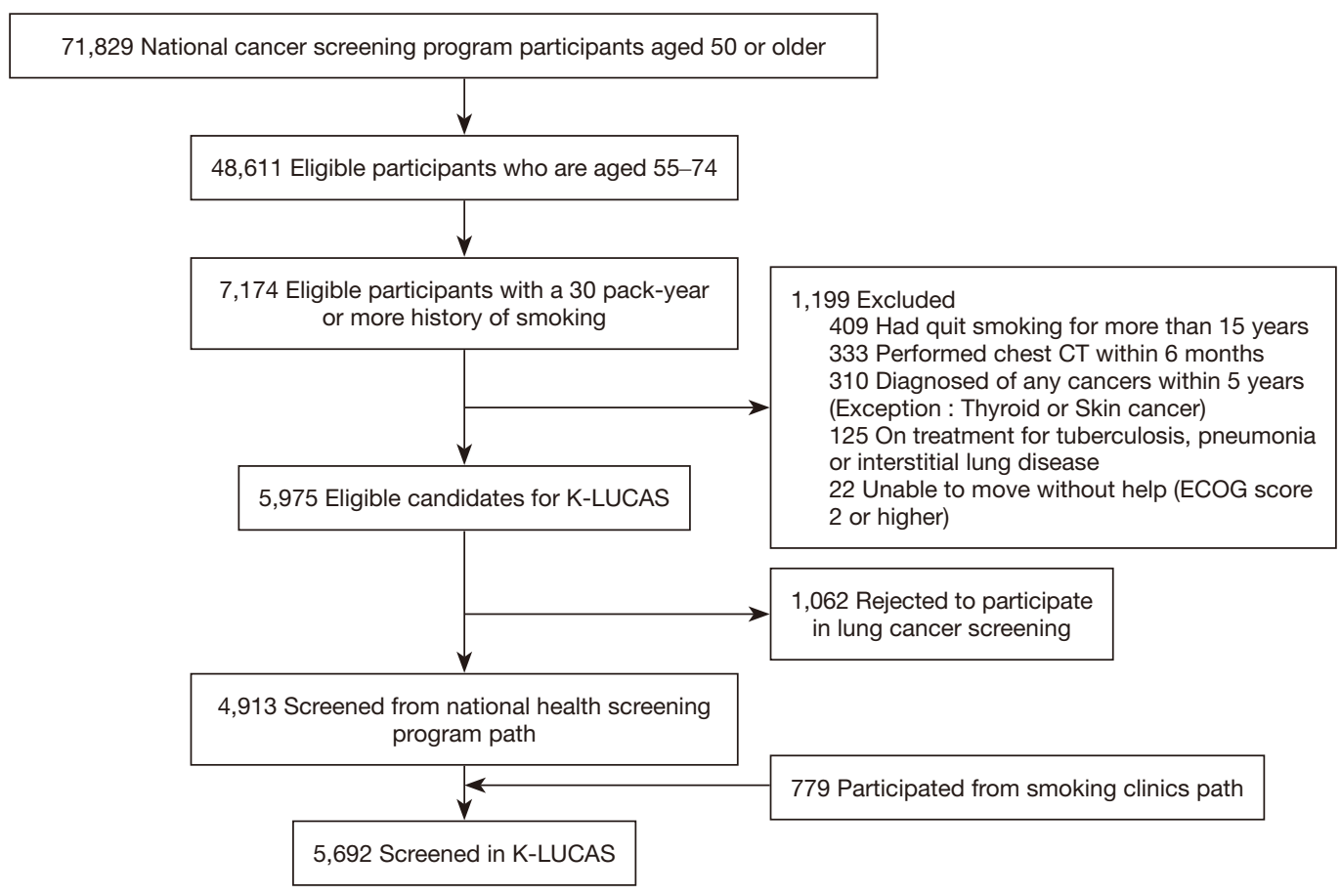

Figure 2 Flow of participant recruitment in Korean Lung Cancer Screening Project (K-LUCAS).

cessation rate was calculated by the percentage of the number of quitters in 6 months after screening by telephone survey among current smoker of participants. All statistical analyses were performed using STATA software ver. 14 (Stata Corp. L.P., College Station, USA).

\section{Results}

\section{Participant characteristics}

Of the 71,829 individuals aged 50 years or older participated in the national health screening program, 5,975 (8.3\%) highrisk individuals were eligible for lung cancer screening. Of 5,975 eligible candidates for K-LUCAS, 1,062 (17.8\%) refused to participate because of no time to visit again for lung cancer screening (52.9\%), no symptom suspected lung cancer (18.6\%), rejection of smoking cessation counselling (7.5\%), radiation harm $(5.1 \%)$, worry about being diagnosed as lung cancer (4.2\%) etc. Thus, the remaining 4,913 highrisk individuals who participated in national health screening program were recruited in K-LUCAS. Additionally, 779 high-risk individuals participated in the smoking cessation program were recruited in K-LUCAS (Figure 2).

A total of 5,692 candidates participated in K-LUCAS between February and December 2017. The participants' characteristics are presented in Table 1. Most of the participants were men, and $53.6 \%$ of them were current smokers. Approximately $23.4 \%$ of the total participants did not finish middle school, and $38.5 \%$ had less than 2.0 million won household income per month. Moreover, $11.3 \%$ of the participants drink alcohols more than 5 days per week, and $40.6 \%$ do not exercise at all. Approximately $10.1 \%$ of the participants had a family history of lung cancer. Tuberculosis was the most common lung disease history among the participants. Approximately $7.2 \%$ of all participants had past tuberculosis treatment history, 3.6\% had chronic obstructive pulmonary disease, and $2.0 \%$ had pneumonia. Some data regarding education level, household income, drinking and aerobic exercise were missing because participants did not answer the survey. Missing data were excluded from analysis.

\section{Effect of advertisement to recruit high-risk candidates}

The number of participants in K-LUCAS greatly increased after the release of public advertisements to recruit highrisk population for lung cancer screening (Figure 3). The proportion of participants with older age, 40 pack-years 
Table 1 General characteristics of participants compared before and after public advertisement ${ }^{\mathrm{a}}$

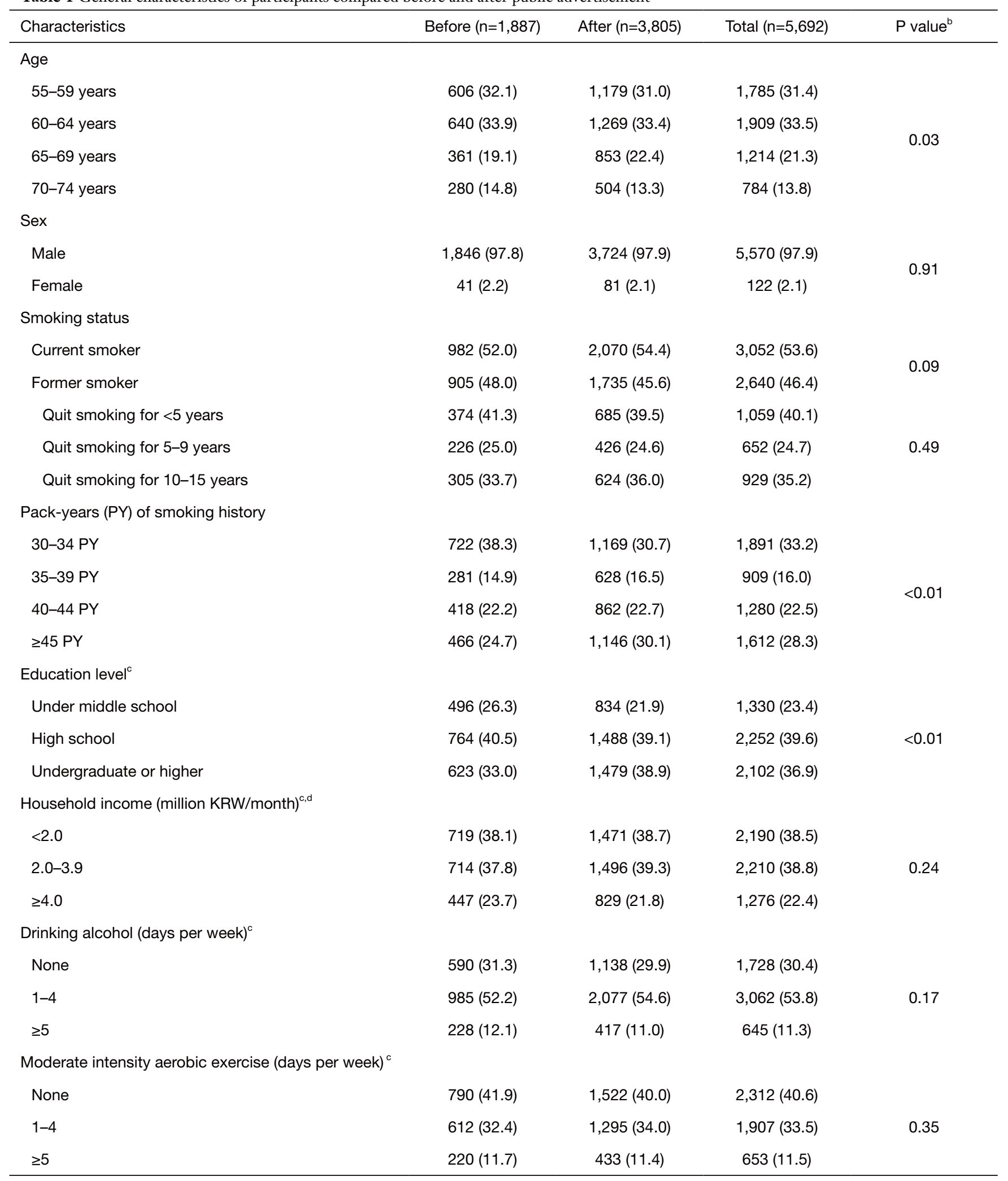

Table 1 (continued) 
Table 1 (continued)

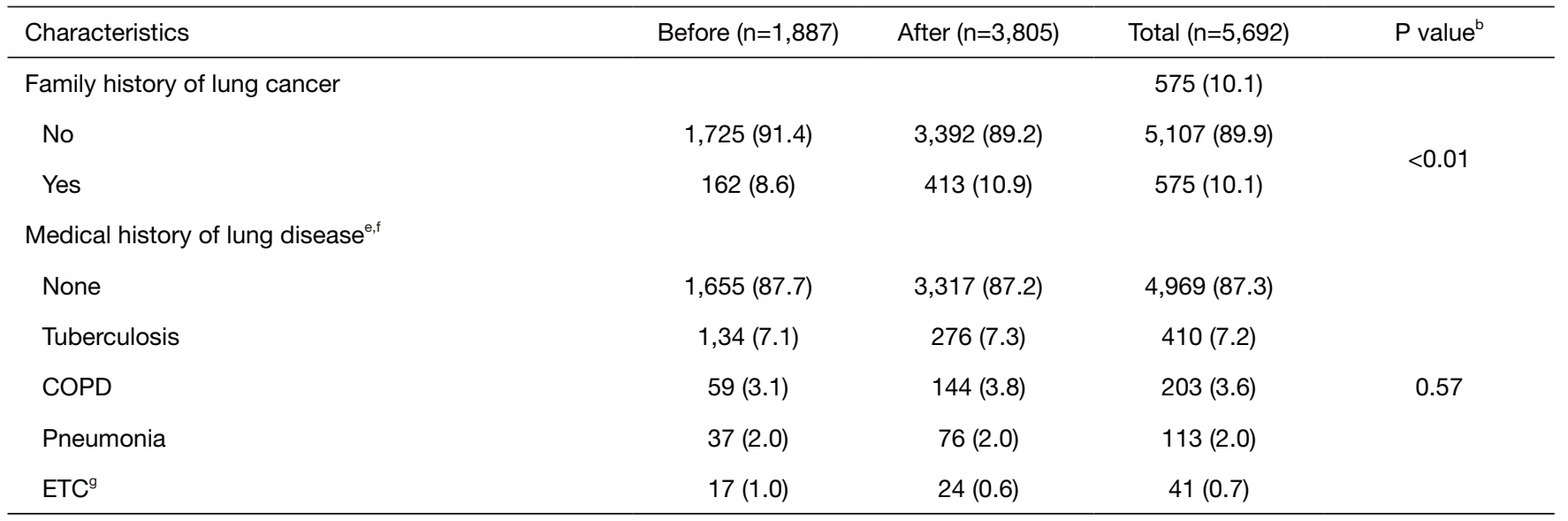

K-LUCAS, Korean Lung Cancer Screening Project; KRW, Korean won; COPD, chronic obstructive pulmonary disease; ARDS, acute respiratory distress syndrome. ${ }^{a}$, advertisement (newspaper) to general population was published on the September 22, 2017, but it generally takes 1 week for the participants to receive the screening. Here, we compared the participants' characteristics before and after October 1, 2017, as we assumed that the advertisement effect began to occur around this date; ${ }^{\mathrm{b}}$, the association between public advertisements and participant characteristics was evaluated using the chi-square test; ${ }^{\circ}$, data missing because the participants did not answer the survey; ${ }^{d}, 1$ million KRW is approximately $900 \mathrm{USD}$; ${ }^{e}$, one participant can develop more than one type of lung disease; ${ }^{f}$, Chi-square test was used to examine the association between public advertisement (before and after advertisement) and participant with any known medical history of lung diseases; ${ }^{9}$, includes solitary nodule, emphysema, fibrothorax, interstitial lung disease, and thickening of pleura.
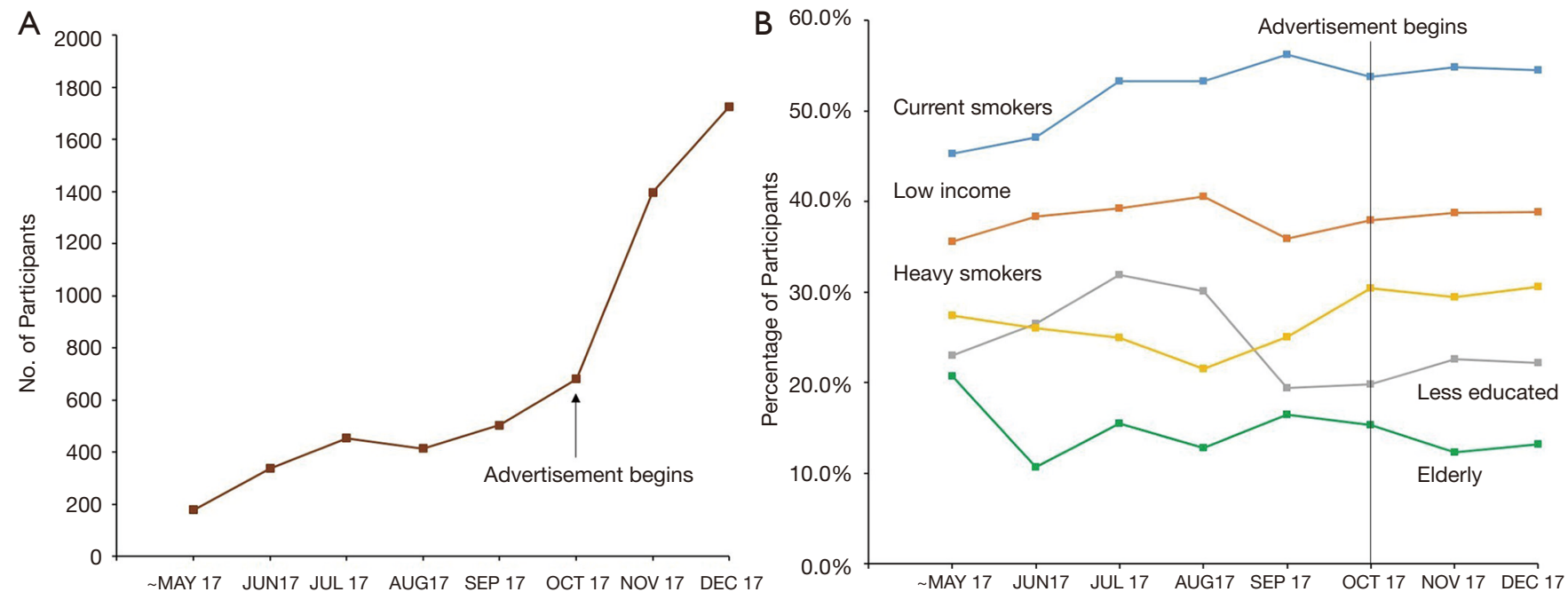

Figure 3 Changes in the number (A) and characteristics (B) of participants over time. (A) shows the changes in the number of participants over time. (B) shows the changes in the characteristics of participants over time. "Current smokers" refer to the percentage of currently smoking participants in each month. "Low income" refers to the percentage of low-income group (monthly household income of less than 2 million KRW). "Heavy smokers" refer to the percentage of participants who have a smoking history of 45 pack-years or more. "Less educated" refers to the percentage of participants who finished secondary education or less, and "smoking clinic path" refers to the percentage of participants from participating smoking clinics. The newspaper advertisement targeting the general population was published on September 22, 2017, but it generally takes 1 week for the participant to undergo screening. Here, we compared the participant characteristics before and after October 1,2017 as we assumed that the effect of the advertisement began around this date. 


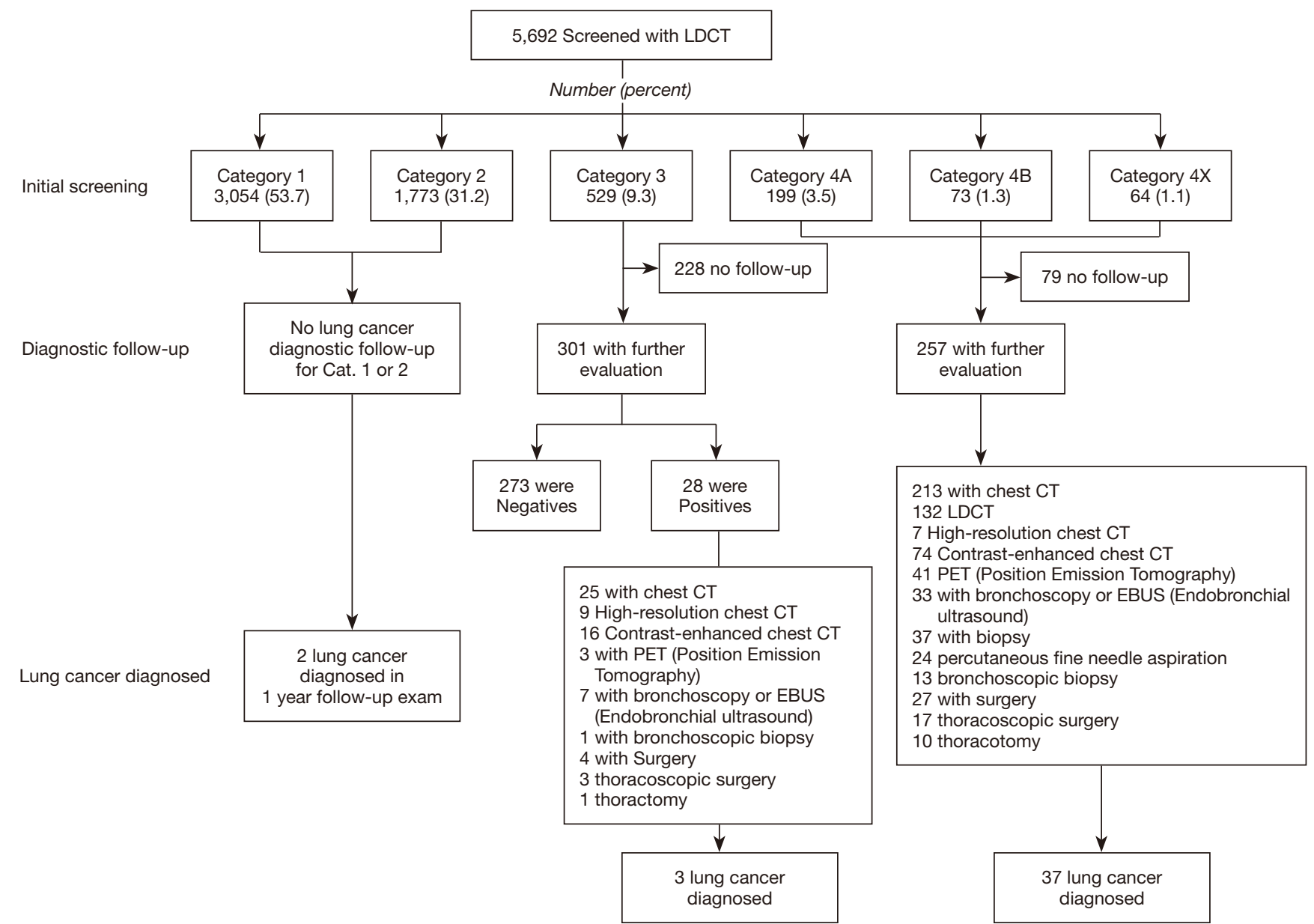

Figure 4 Lung Imaging Reporting and Data System (Lung-RADS) classification of low-dose computer tomography (LDCT) screening and lung cancer diagnostic follow-up procedure. One participant can undergo more than one diagnostic procedure.

or greater smoking history, higher education level, and family history of lung cancer increased after the release of public advertisements. The proportion of former smokers decreased slightly from $48.0 \%$ to $45.6 \%$ after the release of public advertisements.

\section{Screening results}

Among 5,692 participants, 865 (15.2\%) had positive screening results, which requires a further examination; about $9.3 \%$ of the participants had Lung-RADS category 3 , while $5.9 \%$ had category 4 (Figure 4). Of the 529 participants with category 3, 28 exhibited an increase in nodule size on follow-up LDCT. A total of 42 participants were diagnosed with lung cancer. Twenty-four (57.1\%) lung cancer patients were diagnosed with stage I, and four $(9.5 \%)$ with stage II.
During the screening, 37 (88.1\%) lung cancer patients were initially diagnosed with category 4 ; four (7.1\%), category 3 ; and two $(4.8 \%)$, category 2 . Among 865 positive findings (category 3 and 4 ), only 40 cases (4.6\%) were diagnosed as lung cancer. Thus, false positive rate (false positive cases among participants) was $14.5 \%$ and false negative rate (false negative cases which confirmed as lung cancer in category 1 and 2 in screening) was $0.035 \%$. (Table S1).

\section{Follow-up compliance}

Of the 5,692 participants, 865 (15.2\%) were recommended for a further evaluation after baseline screening. However, 307 (35.5\%) refused or could not be reached after calling them thrice. Of the 529 participants with category 3, 301 (56.9\%) underwent further evaluation. Of the 336 participants with 
Table 2 Level of anxiety associated with lung cancer before and after screening by participant characteristics ${ }^{\mathrm{a}}$

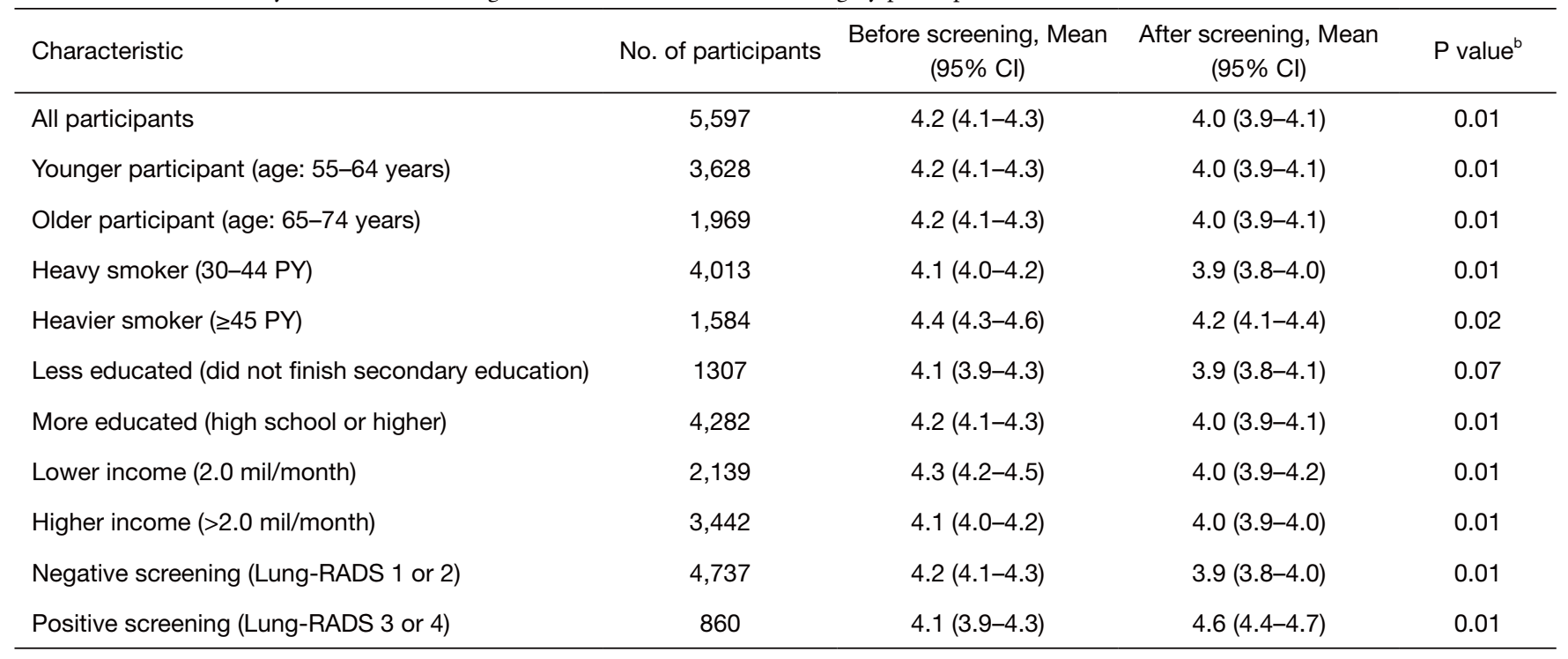

a, Participant's level of anxiety from having lung cancer was subjectively measured on a 0-10 scale, where 0 indicates no anxiety, while 10 indicates the highest degree of anxiety. A total of 95 participants did not answer this particular question in the survey. We only reported the results of the remaining 5,597 participants; ${ }^{b}$, P values were evaluated using a paired t-test. Cl, confidence interval; Lung-RADS, Lung Imaging Reporting and Data System; PY, pack-years.

category 4, 257 (76.5\%) underwent further examination.

\section{Further evaluation and diagnosis}

For further evaluation of positive cases, enhanced or highresolution chest $\mathrm{CT}$ was the most frequent diagnostic procedure, followed by bronchoscopy, needle biopsy, and surgery. After undergoing 36 biopsy procedures, 24 (66.7\%) patients were diagnosed with cancer. Among the 31 patients who underwent surgery, 27 (87.1\%) were diagnosed with cancer. One participant with category 3 was followed up for 15 months until diagnosis of lung cancer.

\section{Complications}

During the diagnostic process, six participants developed complications. Four participants had pneumothorax after percutaneous needle biopsy, and one had excessive bleeding after undergoing thoracoscopy. One participant died after surgery. This participant who was initially diagnosed with category 2 showed nodule enlargement at 1-year followup and was finally diagnosed with lung cancer stage $1 \mathrm{~A}$. This participant developed aspiration pneumonia after undergoing video-assisted lobectomy, which was inferred as the cause of death.

\section{Psychological anxiety}

Psychological anxiety associated with having lung cancer significantly diminished after screening. Only those with positive results were more anxious about having lung cancer after screening (Table 2).

\section{Impact on smoking cessation}

Motivation to quit smoking increased by $9.2 \%$ after participating in the lung cancer screening. The results of the subgroup analysis of participant characteristics and screening results were robust (Table 3). The smoking cessation rate was $24.7 \%$ at 6 -month follow-up, which was determined by telephone survey.

\section{Discussion}

This report provided promising evidence supporting the implementation of a national lung cancer screening program. Previous studies have shown that lung cancer screening conducted in high-risk group using LDCT is an effective method to reduce lung cancer mortality. However, the effectiveness uncertain if it was implemented on a national scale $(10,11)$. Further study outside the clinical 
Table 3 Changes in the level of motivation to quit smoking before and after lung cancer screening by participant characteristics ${ }^{\mathrm{a}}$

\begin{tabular}{|c|c|c|c|c|}
\hline Characteristic & No. of participants & $\begin{array}{l}\text { Before screening, Mean } \\
\qquad(95 \% \mathrm{Cl})\end{array}$ & $\begin{array}{l}\text { After screening, Mean } \\
\qquad(95 \% \mathrm{Cl})\end{array}$ & $P$ value \\
\hline All participants & 2,593 & $6.5(6.4-6.6)$ & $7.1(7.0-7.2)$ & 0.01 \\
\hline Younger participant (age: 55-64 years) & 1,847 & $6.4(6.3-6.5)$ & $7.0(6.9-7.2)$ & 0.01 \\
\hline Older participant (age; 65-74 years) & 746 & $6.6(6.4-6.8)$ & $7.2(7.0-7.4)$ & 0.01 \\
\hline Less educated (did not finish secondary education) & 617 & $6.2(6.0-6.4)$ & $6.9(6.7-7.1)$ & 0.01 \\
\hline More educated (high school or higher) & 1,972 & $6.6(6.5-6.7)$ & $7.2(7.1-7.3)$ & 0.01 \\
\hline Lower income (2.0 mil/month) & 983 & $6.5(6.4-6.7)$ & $7.2(7.0-7.3)$ & 0.01 \\
\hline
\end{tabular}

$\mathrm{Cl}$, confidence interval; Lung-RADS, Lung Imaging Reporting and Data System; PY, pack-years. ${ }^{\text {a }}$, Participant's motivation to quit smoking was subjectively measured on a scale of $0-10$, where 0 indicates no intention to quit smoking and 10 indicates the highest degree of motivation to quit smoking. There were 3,052 current smokers, but 459 did not answer these particular questions in the survey. Hence, we only reported the results of the remaining 2,593 participants. ${ }^{b}, \mathrm{P}$ values were evaluated using a paired t-test.

trials level is may be necessary to evaluate feasibility of implementing a nationwide population-based lung cancer screening program (12).

First, we evaluated the capability to select eligible highrisk participants. In a population-based organized lung cancer screening program, invitation letters are only sent to the eligible high-risk population. In order to select the eligible high-risk population for lung cancer screening, a set of documents that include data on demographics, smoking history, personal and family history of cancer, and medical history are required. These data have been electronically accumulated into the National Health Insurance Service database from the questionnaires collected in the national health screening program in Korea.

However, a questionnaire-based participant selection opens up an opportunity for non-target low-risk population to participate in screening when offered free or at a low cost because one can easily deceive their smoking history on questionnaires. All current smokers underwent the urine cotinine test to verify their current smoking status, but there was no formal test for former smokers. If a non-target low-risk individual was willing to participate in screening, it is most likely that they would be enrolled in the study as a former smoker.

K-LUCAS tested whether publicly available information on free lung cancer screening enrolment would cause non-target-population participation by comparing the participants' characteristics before and after the release of public advertisements. Contrary to what we were worried about, the proportion of higher smoking history population and current smokers were increased after public advertisement to participate in K-LUCAS. We presumed that providing balanced information, including harms of screening, will prevent the participation of low-risk individuals who cannot be verified by cotinine test.

On the other hand, among 5,975 eligible high-risk population for lung cancer screening, 1,062 (17.8\%) rejected to participate in lung cancer screening. The main reasons were they had no time to visit hospital for lung cancer screening, no symptom suspected lung cancer and worry about radiation exposure by CT scan. Therefore, appropriate and balanced information of screening benefits and harms for high-risk population is very important to increase eligible population participation in lung cancer screening. The evidence of lung cancer screening effectiveness for low-risk group of lung cancer is still insufficient. However, private health screening, which is popular among high income group in Korea, provides low dose CT for lung cancer screening to low-risk individuals. Nevertheless, the proportion of exclusion due to recent 
Table 4 Key screening performance of K-LUCAS compared with NLST

\begin{tabular}{lcc}
\hline Indicators & K-LUCAS & NLST \\
\hline True positive & 40 & 270 \\
False positive & 825 & 6,921 \\
False positive rate (\%) & 14.6 & 26.6 \\
Non-cancer case of total positives (\%) & 95.4 & 96.2 \\
Cancer detection rate (\%) & 0.7 & 1.0 \\
TNM stage I detection rate (\%) & 57.1 & 49.1 \\
TNM stage II detection rate (\%) & 9.5 & 6.9 \\
Biopsy procedure rate (\%) & 6.8 & 2.4 \\
Cancer detection per biopsy (\%) & 63.9 & 33.3 \\
Bronchoscopy procedure rate (\%) & 7.2 & 4.8 \\
Cancer detection per bronchoscopy (\%) & 45.0 & 25.1 \\
Surgical procedure rate (\%) & 5.6 & 4.7 \\
Cancer detection per surgical \\
procedure (\%) \\
$\begin{array}{l}\text { Complication per total diagnostic } \\
\text { follow-ups (\%) }\end{array}$ & 87.1 & 75.6 \\
\hline K-LUCAS, Korean Lung Cancer Screening Project & NLST,
\end{tabular}

K-LUCAS, Korean Lung Cancer Screening Project; NLST, National Lung Cancer Trial. Positive findings are defined as presence of nodule with $>6 \mathrm{~mm}$ in diameter, equivalent to Lung-RADS category 3 or 4 . Biopsy procedure rate, cancer detection per biopsy, bronchoscopy procedure rate, cancer detection per bronchoscopy, surgical procedure rate, and cancer detection per surgical procedure were evaluated based on the results of diagnostic follow-ups conducted in patients with categories 3 and 4 only.

receiving chest CT scan among eligible candidates for lung cancer screening is very low about $4.6 \%$ (333 of 7,174). This fact means high risk population for lung cancer is mostly low income group. Thus, it is hard for the high-risk population to have the opportunity to participate in lung cancer screening by high-cost private screening program.

Second, we evaluated if it is feasible to improve screening efficiency if screening would be extended to a national program in Korea. We specifically focused on whether we could improve the early lung cancer detection and smoking cessation compared with other randomized controlled trials.

Of the 42 patients diagnosed with lung cancer, 24 (57.1\%) had TNM stage I and four (9.5\%), stage II. The detection rates of both stage I and stage II reported in K-LUCAS were higher than those in NLST. The detection rates of stage I and stage II reported in NLST were $50.0 \%$ and
$7.1 \%$, respectively. The early-stage detection rates reported up to date in K-LUCAS was also higher than the general early-stage lung cancer detection rates in Korea (20\%). The cancer detection rate $(0.7 \%)$ was, however, lower than that in NLST, probably because the crude lung cancer incidence rate in Korea is 56.4 per 100,000 people, which is lower than that in the United States (69.6 per 100,000 people) (13).

Recent evidence showed that lung cancer screening can be perceived as an alternative to smoking cessation. To help prevent lung cancer, mandatory smoking cessation counseling was provided to all currently smoking participants (14). The motivation to quit smoking has significantly increased after participating in lung cancer screening, which resulted in high smoking cessation rates. The full discussion of the smoking intervention in K-LUCAS will be reported separately in the future. In brief, the cessation rates of smoking at 6-month follow-up was $24.7 \%$, which was higher than that reported in other screening trials (15-17).

Third, we evaluated if it is feasible to reduce the screening harm if the population-based lung cancer screening program was implemented, even in Korea, where tuberculosis is more prevalent than in Western countries (18). We focused on our capability to reduce the false positives and this was compared with that of previous studies. A higher tuberculosis incidence can cause a higher false-positive rates as pulmonary nodules cannot be clearly distinguished from tuberculosis granulomas or scars. Hence, the possibility of reducing the positive rates and falsepositive findings should be assessed as high false-positive rates can increase the likelihood of harm to the participant as well as the cost of lung cancer screening.

The positive and false-positive rates reported were lower than those reported in NLST. K-LUCAS reported a positive rate of $15.2 \%$ and a false-positive rate of $14.6 \%$, whereas NLST reported a positive rate of $27.3 \%$ and a false-positive rate of $26.6 \%$ after the initial screening (Table 4).

The positive rates were, however, remained higher than those reported in another comparable large lung cancer screening trial in Europe (NELSON). The initial positive and false-positive rates in the NELSON trial were $2.6 \%$ and $1.7 \%$, respectively. The key difference was the method of nodule measurement. NLST and K-LUCAS used diameter, whereas the NELSON trial used volume and growth. Although volume and growth measurements seemed superior initially, a 3-to-4-month follow-up (indeterminate category) was performed in the NELSON trial before classifying the cases as positive cases. In the 
NELSON study, $19.4 \%$ of the participants were categorized as indeterminate in the first round of screening, and only $5.3 \%$ were categorized as positive cases. Upon considering all indeterminate results as positive results, the positive and false-positive rates increased to $20.8 \%$ and $20.0 \%$, respectively, in the NELSON study, which were higher than those reported in K-LUCAS. Using the definition of positive findings provided in the NELSON study, which regarded indeterminate findings as positives only if there is a significant change in nodule size at follow-up, the positive and false positive rates in K-LUCAS were $6.7 \%$ and $6.0 \%$, respectively.

Reduction in the positive and false-positive rates prevented the performance of unnecessary invasive diagnostic procedures. The cancer detection rate per biopsy was $63.9 \%$, which is higher than that of NLST (33.3\%). The cancer detection rate per surgical procedure was $87.1 \%$, which was also higher than that of NLST (75.6\%). The complications per total diagnostic follow-ups were lower in K-LUCAS (1.1\%) than in NLST (3.4\%) (Table 4).

However, the low compliance to follow-up examinations after exhibiting positive results to baseline screening was a worrisome issue. In this study, the average follow-up compliance rate was $64.5 \%$, which is substantially lower than that of NLST. Our results imply that high followup compliance rates that have been previously shown in randomized controlled trials may not be achieved in a population-based program. Some participants rejected further examinations even when they received a report of abnormal findings from screening, because they had no symptoms, no time or economic burden for more medical examinations after free screening. Low compliance of further evaluation for positive findings from screening, which was revealed in the current population-based feasibility study, is a really important problem because of losing the chances of detecting early lung cancers with high probability by screening. Strategies to improve follow-up compliance should be considered as this could affect the effectiveness of lung cancer screening.

Furthermore, psychological anxiety associated with having lung cancer significantly diminished after screening except in those participants with positive results (19). Hence, strategies to reduce the anxiety level of participants with positive screening results should be considered.

Fourth, we evaluated the capability to control the screening quality. K-LUCAS has adopted two systematic approaches to maintain the screening quality. First, the LDCT screening results were evaluated by radiologists using the standardized Lung-RADS. The Lung-RADS is used to classify lung nodules by their characteristics (solid, sub-solid, or ground glass) and diameter size. Lung-RADS is more practical than the volumetric nodule management (NODCAT) used in European lung cancer trials on the scope of a national cancer screening as NODCAT requires a follow up scan to measure volume doubling time (VDT). Due to legal restrictions in Korea, a direct transfer of LDCT scan from one hospital to another is not feasible. In order to measure VDT, a participant would be forced to either receive screening twice within the same hospital or carry their own imaging results to another hospital. LungRADS was therefore preferred over NODCAT.

Analysis of sensitivity and specificity in K-LUCAS may require longer follow-up periods. However, on the basis of false-positive rates, the application of Lung-RADS seemed feasible. Most of the patients with confirmed lung cancer were initially diagnosed with category 4 (88.1\%) and category $3(7.1 \%)$. On the other hands, two cases of lung cancer were detected in category 2 . Thus, high-risk population who had negative results from lung cancer screening still need to participate in long-term screening on a regular basis although the one-year screening interval requires further studies (20).

In most countries, only a few radiologists specialize in chest radiography (21). Therefore, implementation of a population-based lung cancer screening would either increase their workload, which would cause quality control problems or increase the patients' waiting time for screening. This was the reason why the networkbased diagnosis supporting system using a CAD program for implementation was established in K-LUCAS. The network-based supporting system aims to minimize diagnostic errors and maximize lung nodule detection sensitivity. It could also help bridge the gap in diagnostic accuracy between a chest specialist and non-chest specialist. In brief, the network-based diagnosis supporting system using CAD increased the positive findings but reduced the variance of positive findings between screening units.

Lastly, with regard to level of infrastructures, there are about 340 general hospitals designated for national health screening programs are equipped with both CT scanners with at least 16 channels and multidisciplinary specialists for lung cancer screening including radiologists in Korea. Based on the results of K-LUCAS, a population-based lung cancer screening program is expected to be implemented in Korea in 2019.

The primary limitation of this study is that we only 
reported interim results of K-LUCAS for short followup period; thus, the mortality rate was not evaluated. Even though, the interim result of lung cancer screening targeting high-risk population is promising in Korea to detect early stage lung cancer and reduce false positive rate compared to randomized trials conducted in western countries by using quality control network system, still some problems are remained including rejection of participating in screening among high-risk individuals and low compliance for further diagnostic process among screening participants having abnormal results. Further evaluation on the feasibility of implementing populationbased lung cancer screening with follow-up data would be reported in the future.

\section{Acknowledgments}

We thank all K-LUCAS trial investigators including Dr. Mina Suh (National Cancer Center), Dr. Sook Whan Sung (Catholic University of Korea), Dr. Hong Kwan Kim (Samsung Medical Center), Dr. Chang-Min Choi (Asan Medical Center), Dr. Young-Kin Tak (Busan Regional Cancer Center), Dr. A-Sol Kim (Daegu-Gyeongbuk Regional Cancer Center), Dr. Seok-Joon Yoon (Daejeon Regional Cancer Center), Dr. Woo Jin Kim (Gangwon Cancer Center), Dr. Seung Su Sin (Gyeonggi Cancer Center), Dr. Sang Pyo Lee (Incheon Regional Cancer Center), Dr. Jung-Sik Huh (Jeju Regional Cancer Center), Dr. Gong-Young Jin (Jeonbok Regional Cancer Center), Dr. Young-Chul Kim (Jeonnam Regional Cancer Center), Dr. Seon-Mee Kim (Korea University Guro Hospital), Dr. Seung Hyeun Lee (Kyunghee University Hospital), Dr. Chang-Hoon Lee (Seoul National University Hospital), and Dr. Seung Won Ra (Ulsan Cancer Center).

Funding: This study was supported by grants (1631000 and 1720310) from the National R\&D Program for Cancer Control, Ministry of Health and Welfare, Republic of Korea, and national cancer center grant (1610430), National Cancer Center, Republic of Korea.

\section{Footnote}

Reporting Checklist: The authors have completed the STROBE reporting checklist. Available at http://dx.doi. org/10.21037/tlcr-20-700

Data Sharing Statement: Available at http://dx.doi. org/10.21037/tlcr-20-700
Conflicts of Interest: All authors have completed the ICMJE uniform disclosure form (available at http://dx.doi. org/10.21037/tlcr-20-700). The authors have no conflicts of interest to declare.

Ethical Statement: The authors are accountable for all aspects of the work in ensuring that questions related to the accuracy or integrity of any part of the work are appropriately investigated and resolved. The study conformed to the provisions of the Declaration of Helsinki (as revised in 2013). K-LUCAS was approved by the institutional review board of each participating hospital. An informed consent form was obtained from all participants after providing them with information regarding the benefits and harms of lung cancer screening.

Open Access Statement: This is an Open Access article distributed in accordance with the Creative Commons Attribution-NonCommercial-NoDerivs 4.0 International License (CC BY-NC-ND 4.0), which permits the noncommercial replication and distribution of the article with the strict proviso that no changes or edits are made and the original work is properly cited (including links to both the formal publication through the relevant DOI and the license). See: https://creativecommons.org/licenses/by-nc-nd/4.0/.

\section{References}

1. Allemani C, Matsuda T, Di Carlo V, et al. Global surveillance of trends in cancer survival: analysis of individual records for 37,513,025 patients diagnosed with one of 18 cancers during 2000-2014 from 322 populationbased registries in 71 countries (CONCORD-3). Lancet 2018;391:1023-75.

2. Jung KW, Won YJ, Kong HJ, et al. Cancer Statistics in Korea: Incidence, Mortality, Survival, and Prevalence in 2016. Cancer Res Treat 2019;51:417-30.

3. National Lung Screening Trial Research Team, Aberle DR, Adams AM, et al. Reduced lung-cancer mortality with low-dose computed tomographic screening. N Engl J Med 2011;365:395-409.

4. van Klaveren RJ, Oudkerk M, Prokop M, et al. Management of lung nodules detected by volume CT scanning. N Engl J Med 2009;361:2221-9.

5. Brierley JD, Gospodarowicz MK, Wittekind Ch. TNM classification of malignant tumors (8th ed). WileyBlackwell, 2017.

6. American College of Radiology [Internet]. Reston: 
Lung Imaging Reporting and Data System (Lung-RAD) [cited 2018 June 18]. Available online: https://www.acr. org/Clinical-Resources/Reporting-and-Data-Systems/ Lung-Rads

7. McKee BJ, Regis SM, McKee AB, et al. Performance of ACR Lung-RADS in a clinical CT lung screening program. J Am Coll Radiol 2015;12:273-6.

8. Lee J, Lim J, Kim Y, et al. Development of protocol for Korean Lung Cancer Screening Project (K-LUCAS) to evaluate effectiveness and feasibility to implement national cancer screening program. Cancer Res Treat 2019;51:1285-94.

9. Lee JW, Kim HY, Goo JM, et al. Radiological report of pilot study for the Korean Lung Cancer Screening (K-LUCAS) project: feasibility of implementing Lung Imaging Reporting and Data System. Korean J Radiol 2018;19;803-8.

10. Prosch H. Implementation of lung cancer screening: promises and hurdles. Transl Lung Cancer Res 2014;3:286-90.

11. Optican RJ, Chiles C. Implementing lung cancer screening in the real world: opportunity, challenges and solutions. Transl Lung Cancer Res 2015;4:353-64.

12. Shankar A, Saini D, Dubey A, et al. Feasibility of lung cancer screening in developing countries: challenges, opportunities and way forward. Transl Lung Cancer Res 2019;8:S106-21.

13. Forman D, Bray F, Brewster DH, et al. Cancer Incidence in five continents, Vol.X. IARC Scientific Publication No.

Cite this article as: Lee J, Kim Y, Kim HY, Goo JM, Lim J, Lee CT, Jang SH, Lee WC, Lee CW, Choi KS, Park B, Lee DH; for the K-LUCAS Trial Investigators. Feasibility of implementing a national lung cancer screening program: Interim results from the Korean Lung Cancer Screening Project (K-LUCAS). Transl Lung Cancer Res 2021;10(2):723736. doi: 10.21037/tlcr-20-700
164. Lyon: International Agency for Research on Cancer.

14. Taylor KL, Cox LS, Zincke N, et al. Lung cancer screening as a teachable moment for smoking cessation. Lung Cancer 2007;56:125-34.

15. Ashraf H, Tønnesen P, Holst Pedersen J, et al. Effect of CT screening on smoking habits at 1-year follow-up in the Danish Lung Cancer Screening Trial (DLCST). Thorax 2009;64:388-92.

16. Brain K, Carter B, Lifford KJ, et al. Impact of low-dose CT screening on smoking cessation among high-risk participants in the UK Lung Cancer Screening Trial. Thorax 2017;72:912-8.

17. van der Aalst CM, van Klaveren RJ, van den Bergh KA, et al. The impact of a lung cancer computed tomography screening result on smoking abstinence. Eur Respir J 2011;37:1466-73.

18. World Health Organization [Internet]. Global tuberculosis report 2017. [cited 2018 June 18]. Available online: https:// www.who.int/tb/publications/global_report/en

19. Bach PB, Mirkin JN, Oliver TK, et al. Benefits and harms of CT screening for lung cancer: a systematic review. JAMA 2012;307:2418-29.

20. Ruano-Ravina A, Pérez-Ríos M, Casàn-Clará $\mathrm{P}$, et al. Low-dose CT for lung cancer screening. Lancet Oncol 2018;19:e131-2.

21. The Society of Radiographers [Internet]. The radiography workforce current challenges and changing needs. 2016. [cited 2018 June 18]. Available online: https://www.sor. org/sites/default/files/document-versions/appg_a4.pdf 
Table S1 Lung cancer diagnosed in K-LUCAS (Case review)

\begin{tabular}{|c|c|c|c|c|c|c|c|c|}
\hline \multirow{2}{*}{ Case } & \multirow{2}{*}{ Sex } & \multirow{2}{*}{ Age } & \multirow{2}{*}{ Lung-RADS } & \multirow{2}{*}{ Diagnosis } & \multirow{2}{*}{ TNM $\left(8^{\text {th }}\right)$} & \multicolumn{3}{|c|}{ Treatment } \\
\hline & & & & & & Surgery & Radiotherapy & Chemotherapy \\
\hline 1 & M & 74 & $4 \mathrm{~A}$ & Small cell cancer & IIIC & N & N & Y \\
\hline 2 & M & 72 & $4 \mathrm{X}$ & Large cell neuroendocrine carcinoma & IB & $\mathrm{Y}$ & $\mathrm{N}$ & $\mathrm{Y}$ \\
\hline 3 & M & 72 & $4 X$ & Adenocarcinoma & IA1 & Y & N & N \\
\hline 4 & M & 74 & $4 \mathrm{X}$ & Squamous cell carcinoma & IIA & Y & N & $\mathrm{N}$ \\
\hline 5 & M & 60 & $4 \mathrm{X}$ & Adenocarcinoma & IA2 & $\mathrm{Y}$ & $\mathrm{N}$ & N \\
\hline 6 & M & 70 & $4 \mathrm{X}$ & Adenocarcinoma & IIIB & $\mathrm{N}$ & Y & $\mathrm{Y}$ \\
\hline 7 & M & 70 & $4 \mathrm{~B}$ & Adenocarcinoma & IVA & $\mathrm{N}$ & Y & $\mathrm{Y}$ \\
\hline 8 & M & 61 & $4 \mathrm{X}$ & Squamous cell carcinoma & IIIA & Y & Y & $\mathrm{Y}$ \\
\hline 9 & M & 72 & $4 \mathrm{~A}$ & Adenocarcinoma & IB & $\mathrm{Y}$ & $\mathrm{N}$ & $\mathrm{N}$ \\
\hline 10 & M & 72 & $4 \mathrm{X}$ & $\begin{array}{l}\text { Non-small cell cancer, Squamous cell carcinoma } \\
\text { (combined) }\end{array}$ & IIIA & $\mathrm{N}$ & N & $\mathrm{Y}$ \\
\hline 11 & M & 62 & $4 X$ & Adenocarcinoma & IA2 & $\mathrm{Y}$ & $\mathrm{N}$ & N \\
\hline 12 & M & 67 & $4 \mathrm{X}$ & Squamous cell carcinoma & IVB & $\mathrm{N}$ & N & $\mathrm{Y}$ \\
\hline 13 & M & 63 & $4 \mathrm{~B}$ & Squamous cell carcinoma & IIIA & $\mathrm{Y}$ & Y & $\mathrm{Y}$ \\
\hline 14 & M & 64 & $4 \mathrm{X}$ & $\begin{array}{l}\text { Small cell cancer, Squamous cell carcinoma } \\
\text { (combined) }\end{array}$ & IIA & $N$ & Y & $\mathrm{Y}$ \\
\hline 15 & M & 74 & $4 X$ & Squamous cell carcinoma & IIIA & $\mathrm{N}$ & Y & $\mathrm{Y}$ \\
\hline 16 & M & 63 & $4 \mathrm{~B}$ & Squamous cell carcinoma & IVB & $\mathrm{N}$ & Y & $\mathrm{N}$ \\
\hline 17 & M & 61 & $4 \mathrm{~B}$ & Squamous cell carcinoma & IA1 & $\mathrm{Y}$ & Y & $\mathrm{N}$ \\
\hline 18 & M & 62 & $4 \mathrm{~B}$ & Small cell cancer, Adenocarcinoma (combined) & IIB & $\mathrm{Y}$ & N & N \\
\hline 19 & M & 72 & $4 \mathrm{~B}$ & Squamous cell carcinoma & IVB & $\mathrm{N}$ & Y & $\mathrm{Y}$ \\
\hline 20 & M & 69 & $4 \mathrm{~B}$ & Adenocarcinoma & IA2 & $\mathrm{Y}$ & N & N \\
\hline 21 & M & 56 & $4 \mathrm{~B}$ & Adenocarcinoma & IA & Y & N & N \\
\hline 22 & M & 62 & $4 \mathrm{X}$ & Adenocarcinoma & IA2 & Y & N & N \\
\hline 23 & M & 63 & $4 X$ & Non-small cell cancer & IVA & $\mathrm{N}$ & $\mathrm{N}$ & $\mathrm{Y}$ \\
\hline 24 & M & 57 & $4 \mathrm{X}$ & Adenocarcinoma & IB & Y & N & N \\
\hline 25 & M & 55 & $4 \mathrm{~B}$ & Adenocarcinoma & IB & $\mathrm{Y}$ & $\mathrm{N}$ & $\mathrm{N}$ \\
\hline 26 & M & 61 & $4 X$ & Adenocarcinoma & IIB & $\mathrm{Y}$ & N & $\mathrm{Y}$ \\
\hline 27 & M & 74 & $4 X$ & Adenocarcinoma & IIIB & $\mathrm{Y}$ & N & N \\
\hline 28 & M & 55 & $4 \mathrm{~B}$ & Adenocarcinoma & IB & $\mathrm{Y}$ & N & $\mathrm{Y}$ \\
\hline 29 & M & 60 & $4 X$ & Squamous cell carcinoma & IA1 & $\mathrm{Y}$ & Y & $\mathrm{N}$ \\
\hline 30 & M & 58 & $4 \mathrm{~A}$ & Adenocarcinoma & IA1 & $\mathrm{Y}$ & N & $\mathrm{N}$ \\
\hline 31 & $M$ & 65 & $4 X$ & Adenocarcinoma & IB & $\mathrm{Y}$ & N & N \\
\hline 32 & M & 70 & $4 X$ & Adenocarcinoma & IA3 & $\mathrm{Y}$ & N & $\mathrm{N}$ \\
\hline 33 & M & 68 & $4 \mathrm{~B}$ & Adenocarcinoma & IA2 & $\mathrm{Y}$ & N & $\mathrm{N}$ \\
\hline 34 & M & 63 & $4 X$ & Adenocarcinoma & IA2 & $\mathrm{Y}$ & N & $\mathrm{N}$ \\
\hline 35 & M & 65 & $4 X$ & Small cell cancer, & IIIA & $\mathrm{N}$ & $\mathrm{Y}$ & $\mathrm{Y}$ \\
\hline 36 & M & 74 & $4 \mathrm{~B}$ & Adenocarcinoma & IB & $\mathrm{N}$ & Y & N \\
\hline 37 & M & 64 & 3 & Squamous cell carcinoma & IB & $\mathrm{Y}$ & N & Y \\
\hline 38 & M & 62 & 3 & Adenocarcinoma & IB & $\mathrm{Y}$ & N & $\mathrm{Y}$ \\
\hline 39 & M & 62 & $4 \mathrm{~A}$ & Squamous cell carcinoma & IIIB & $N$ & N & N \\
\hline 40 & M & 60 & 2 & Adenocarcinoma & IB & $\mathrm{Y}$ & $\mathrm{N}$ & $\mathrm{N}$ \\
\hline 41 & M & 71 & 2 & Squamous cell carcinoma & IA & Y & N & N \\
\hline 42 & $M$ & 60 & 3 & Adenocarcinoma & IA & $\mathrm{Y}$ & $\mathrm{N}$ & $\mathrm{N}$ \\
\hline
\end{tabular}

K-LUCAS, Korean Lung Cancer Screening Project; Lung-RADS, Lung Imaging Reporting and Data System. 